\title{
Towards Establishing Path Planning Strategies For Autonomous UAVs; A Brief Survey- Summary on Recent Techniques
}

\author{
Anees ul Husnain ${ }^{12}$, Norrima Binti Mokhtar ${ }^{1}$, Noraisyah Binti Mohamed Shah ${ }^{1}$, Mahidzal Bin Dahari ${ }^{1}$ \\ ${ }^{1}$ Department of Electrical Engineering, University of Malaya, Kuala Lumpur, Malaysia \\ ${ }^{2}$ Applied Controls and Robotics Research Laboratory, University of Malaya, Kuala Lumpur, Malaysia \\ (Tel: 60-10-245-5060) \\ norrimamokhtar@um.edu.my
}

\begin{abstract}
The extent of autonomy in path planning for a UAV primarily depends upon the capabilities of its algorithm. The diversity in UAV applications and an abundance of choices in autonomous path planning algorithms are swelling every day, so the selection of most appropriate algorithm gets baffling. The past two decades of research on UAVs revealed that seventy percent of it had been published in the previous three and a half years. Hence, a comprehensive survey study was proposed and conducted to obtain an overview of the recent developments in autonomous path planning applications and their respective algorithms. This article presents a summary of the survey and suggests most suitable path planning algorithms for a UAV application.
\end{abstract}

Keywords: Autonomous UAV, Survey, UAV Path Planning.

\section{Introduction}

UAVs can provide highly useful data, and their role becomes vital in information collection, aerial surveillance, industrial or agricultural monitoring, preventing disasters and so on. However, the most significant element that is common in most of these applications to pick out would be the access to realtime data. The role of UAV systems, due to their cost effectiveness and eased of access to deploy has influentially motivated scientists to opt them as a tremendous support to research Nevertheless, this would require a higher level of autonomous behavior among UAVs.

An autonomous flight correctly points towards an onboard intelligent sensors-based system that is expected to set and modify the intermediate flight paths, to meet the mission objectives. An Unmanned Aerial Vehicle (UAV) may either follow a preprogrammed flight trajectory, a remotely operated path by a human or an dynamic path calculation during the flight.[1] At all the times in flight, a UAV must maintain or observe the parameters that mainly include stable flight dynamics, energy consumptions, mission requirements or to restrain themselves within the areas of interest. This gives a very significant role to the aspect of path planning in UAV operations, as a global objective, alongside the local flight requirements.

Usually, a UAV has a global and local set of dynamics and parameters alongside a high- and lowlevel control strategies, to be generic. Path planning is a matter of higher-level UAV control strategy where the UAV considers global parameters to reach it optimality conditions.

From a broader perspective, path planning can be categorized into two groups, namely offline and online path planning. The path planning before the take-off is offline while modification of flight path during the flight, as required by the mission objectives or the environmental dynamics, is online path planning.

The extent of maximum autonomy that a UAV can achieve is an arbitrarily unachievable as it

primarily depends upon degree of autonomous operations that a UAV may conduct or a group, 
Anees ul Husnain, Norrima Binti Mokhtar, Noraisyah Binti Mohamed Shah, Mahidzal Bin Dahari

swarm and flock of UAV may conduct. It can also be inferred with respect to current developments in this technology that the maximum autonomy of a UAV is a goal still unclear. However, a certainty in this regard so far is that a higher level of autonomy would inevitably require exploiting the onboard computational capabilities that demand computationally intelligent algorithms. Therefore, numerous approaches, criteria and innovations have been observed in effort to enhance the performance of autonomous flights. The graph depicted in fig.1. shows the published literature, indexed by the web of science, for the keyword "UAV" in the past 20 years. It shows that approximately $70 \%$ of the articles, published from 2016 to 2019.[2]

Another important factor to mention is the supporting role of UAVs in multidisciplinary research. This has contributed momentously in the spans of Geology, Chemistry, Environmental Sciences, Agriculture, Material Sciences, Physical Geography, Water Resources, Transportations, Mechanics, and many other research areas, elaborated in fig.2.[7]. Optimistically, this has brought scientists and engineers much closer to several aspects of their research.

\section{Methodology}

The selection of articles was based on two separate queries, i.e., autonomous path planning for UAVs and autonomous UAVs swarm. The search was conducted on the Web of Science Core Collection database for the past five years. From the results, potential groups and subgroups were established. The research undertaken to improve the performance of existing algorithms or introducing innovative ideas for computing algorithms were grouped as developments in autonomous path planning algorithms. The rest of the part was considered as developments in UAV applications.

Algorithms were assigned labels relating to the category of their knowledge streams, as an instance, bio-inspired algorithms, graph search, etc. The algorithms in the second group were further labelled concerning the target application.

\section{Needfulness}

It was required to find the most suited algorithm for a specific target application and to identify a potential set of UAV applications for a particular algorithm. After discovering the top-notch algorithms and applications, an application to algorithms mapping was presented to complete the picture.

Some excellent surveys on path planning algorithms in recent years. In these surveys, the focus was relatively concise in most of them. A few studies about planning algorithms considered workspace dimensions, [3] and obstacle avoidance, [4] where the primarily concern was the nature of workspace and amount of information available to the robot. Another to mention is a technique-specific path computation,[5] moving obstacles cluttered environments.[6]. These dealt with the unexpected changes within the workspace and primarily deal with the lower level control of the UAV.

The considerations for this study were more towards practicality and direct in approach; first, the most recent developments in ongoing autonomous path planning applications and second, the algorithms associated with the most focused UAV applications.

This helped to develop an application to algorithms mapping out of the recent published literature. that offers the reader with appropriate choices of algorithms for a specific UAV application. 


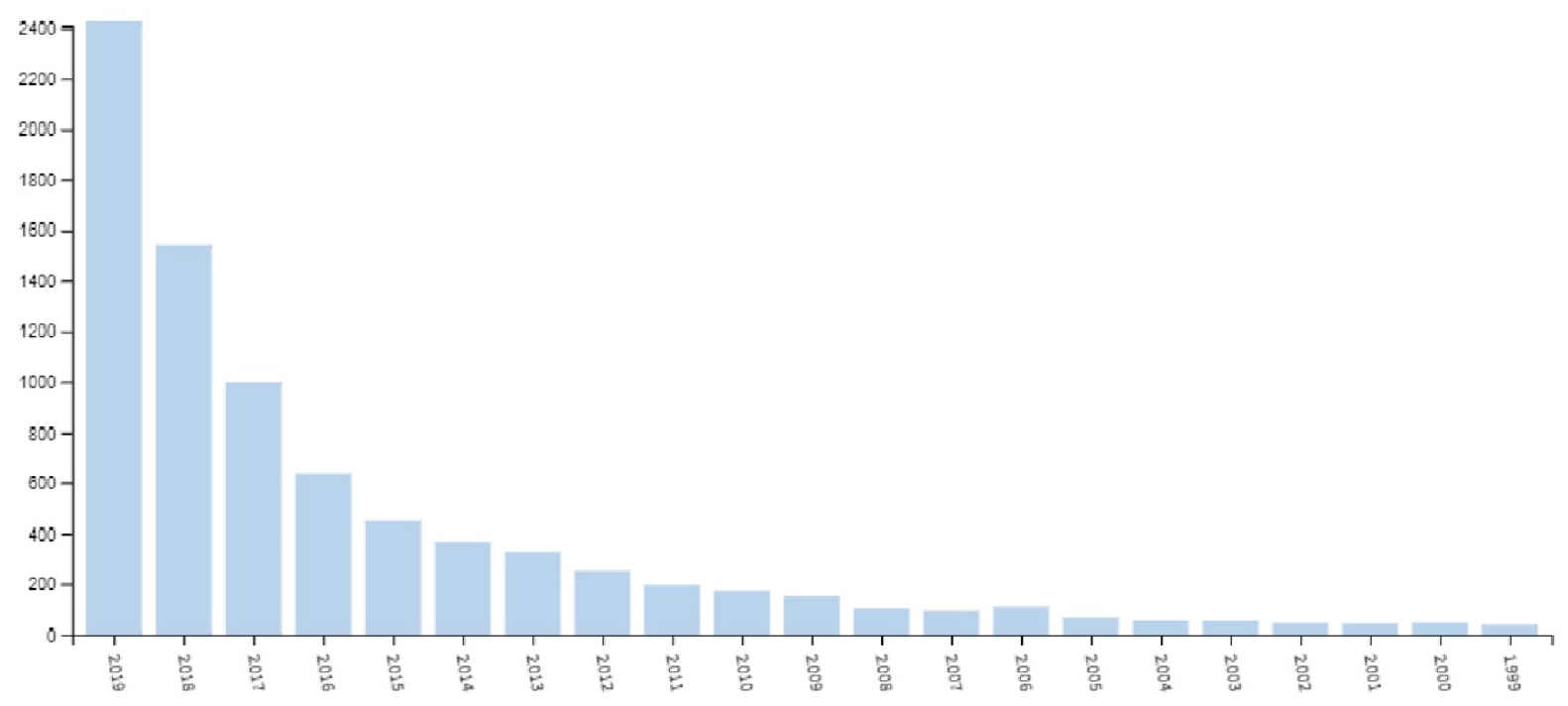

Fig. 1. Growth of research on UAVs in the past two decades (X: years, Y: publications)[2]
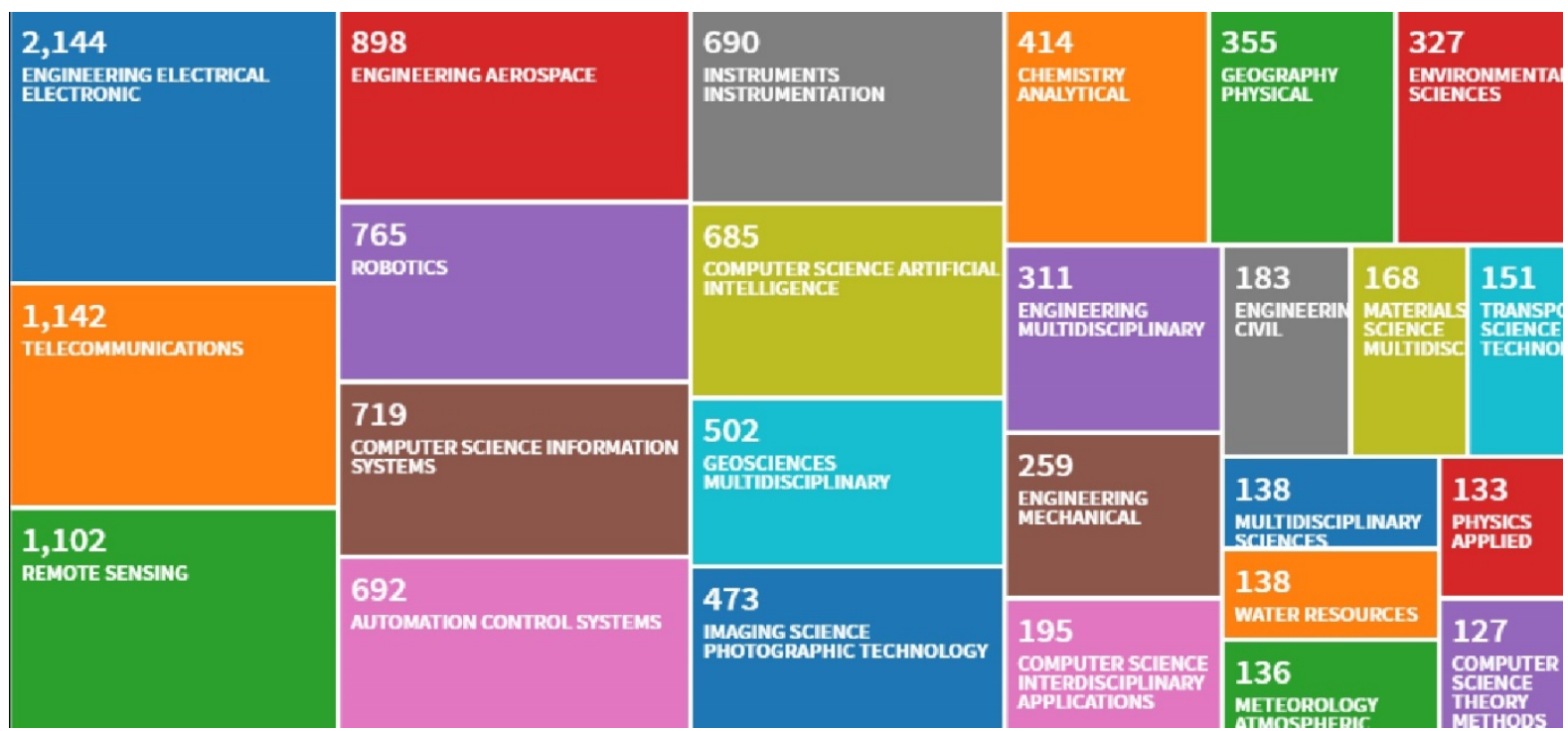

Fig. 2. Contribution of UAVs in Multi-Disciplinary Research Areas (2000-2020)[7]

\section{Survey Findings}

The conducted survey was categorized under two sections; one, most widely utilized algorithms and two, most significant UAV applications. The relation established between these two categories was found helpful in identifying the usefulness of algorithms and promising UAV applications. Hence, identifying the focus of UAV's path planning research and its directions. At here, only the survey findings from the algorithmic developments and most focused applications have been presented, respectively below.

It was found that the fusion of two or more algorithms was $25 \%$ of all the algorithms, and Evolutionary algorithms were widely chosen for the 
Anees ul Husnain, Norrima Binti Mokhtar, Noraisyah Binti Mohamed Shah, Mahidzal Bin Dahari

optimization process. However, the utilization of bio-inspired algorithms and modifications, if combined, these comprised $37 \%$ of the whole pie chart, depicted in Fig. 3.

From the survey, it was also observed that Cooperative Mission planning among UAVs has been the hottest and most focused research area in

Autonomous Path Planning. Their reasons mainly include cost-effective, precision to conduct lower atmospheric aerial operations, ease of deployment, mobility, and accessibility of the required equipment. The second most attractive application was Sense \&

Avoid, followed by extended flight duration and coverage area, applications. New challenges have emerged in UAV research for communication infrastructure among UAVs, a unified identification of foes and friendly flights, secure communications, and UAV traffic regulatory systems.
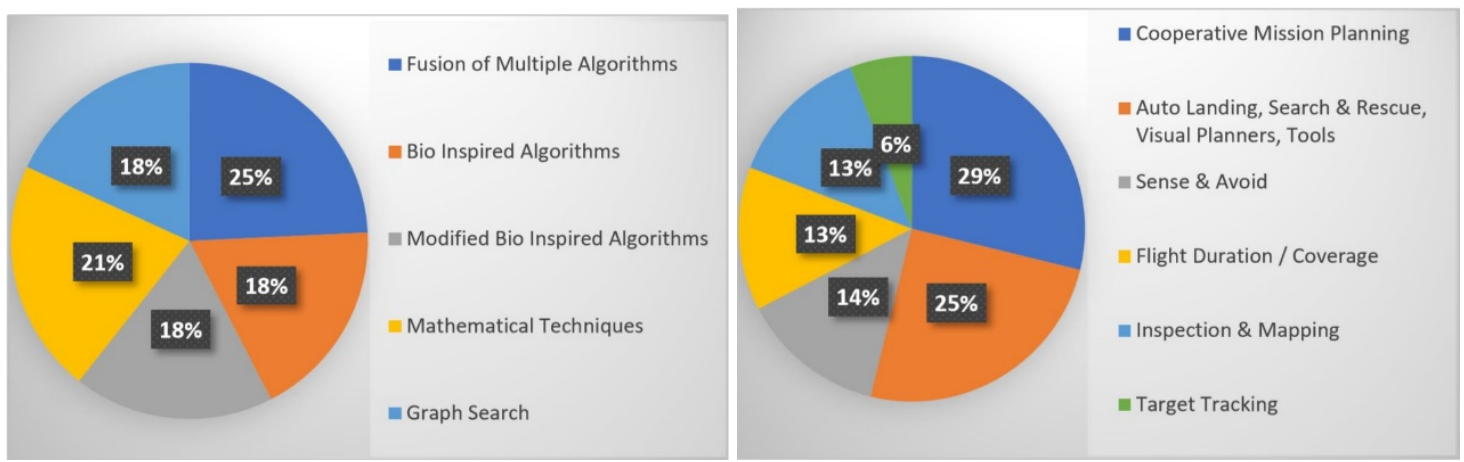

Fig.3. Breakdown of autonomous UAV path planning algorithms (left).and applications (right)

\section{Reflections}

Very high diversity in path planning applications and available algorithms engendered skepticism about the selection of a better or most suited algorithm for a specific UAV application. Therefore, autonomous path planning applications and the algorithms utilized for these have been mapped in Fig. 4. Some of the algorithms can be observed for serving different categories of applications, while others are dedicatedly developed to solve a particular path planning problem. It can also be inferred as the figure illustrates the most focused and demanded features of a UAV that provides a hint of futuristic models. The future of UAVs and developments in computational intelligence would look forward to the inclusion and selection of different data-process models to attain the highest possible intelligence capabilities, under the lights of techniques like reinforcements learning.
However, there have been some worth mentioning observations:

1. It is quite disturbing to notice an absence of Machine Learning techniques

2. there can be observed a huge dominance of optimization algorithms.

3. The highest level of autonomy has not reached, and it stands as a goal not clear yet.

4. Any machine that is supposed to be intelligent must contain a set of specific smart or intelligent actions. The intelligent actions come from intelligent algorithms.

5. A criteria that can be foreseen at this point in time can be as follows: The selection of autonomous UAV's behavior from UAV itself, based on the randomness of its environment and mission objectives, ought to be rated as the highest degree of autonomy and hence may serve as the ultimate test of UAV's autonomy. 


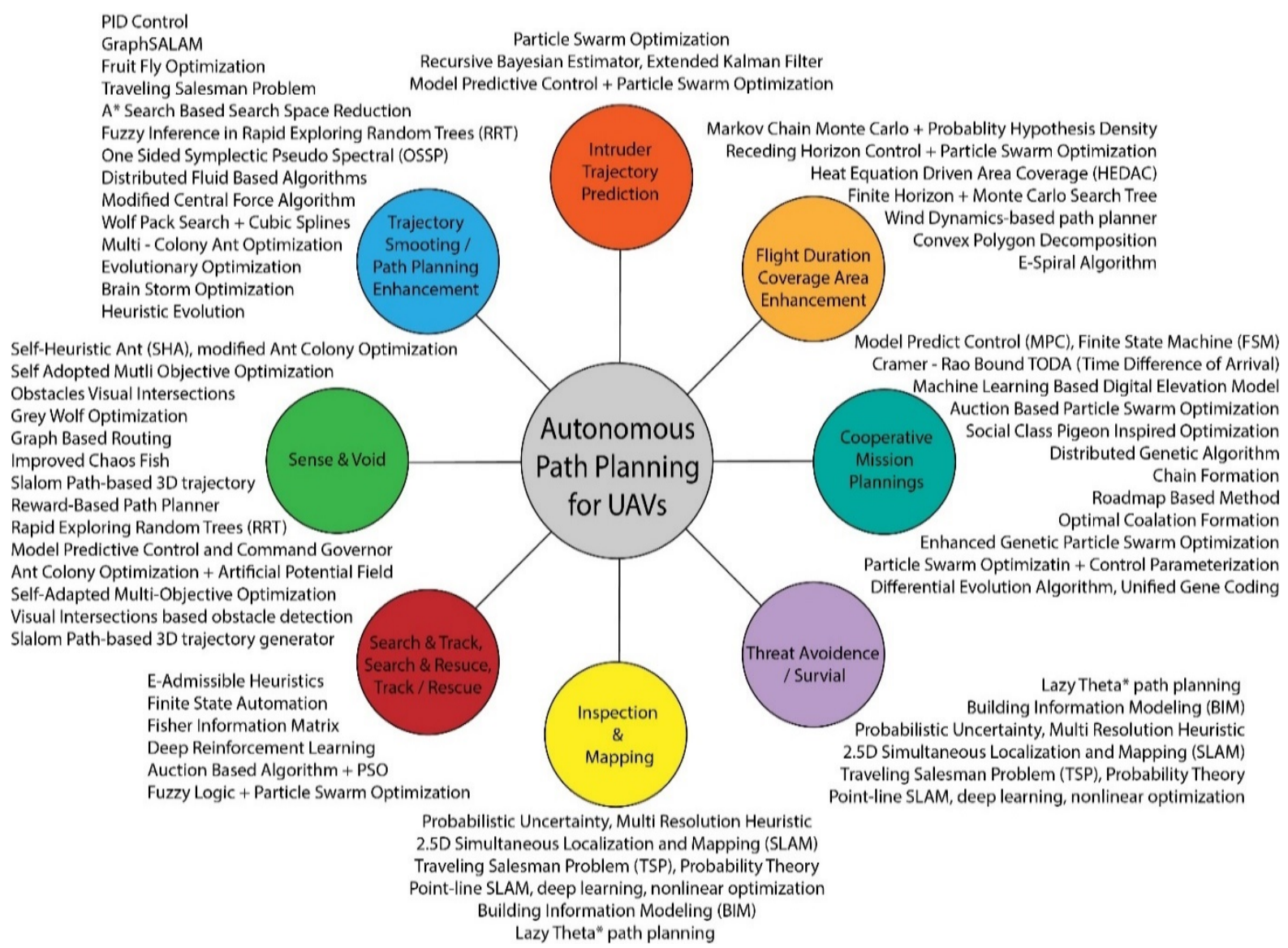

Fig. 4. Applications to Algorithms Mapping for Autonomous Path Planning of UAVs

\section{Acknowledgments:}

The study was carried out as part of the research on "Autonomous Path Planning of UAVs for Environmental Monitoring" at the University of Malaya, Kuala Lumpur, Malaysia. The work is supported by University of Malaya, grant number: GPF062A-2018 for experimental equipment procurement. The Ph.D. scholar is sponsored by a funded scholarship from the Higher Education Commission (HEC) of Pakistan. The author would also like to acknowledge the support from the Islamia University of Bahawalpur, Pakistan.

\section{References:}

1 Cabreira, T.M., L.B. Brisolara, and P.R.F. Jr., Survey on Coverage Path Planning with Unmanned Aerial Vehicles. Drones, 2019. 3(1).
2 Analyzer, C.A.-S.R., 'UAV' search results analysis: Publication Years (1999-2019), in Clarivate Analytics - Web of Science, U.s.r.a.P.Y. (1999-2019), Editor. 2020, Clarivate Analytics: Online.

3 Yang, L., et al., A literature review of UAV 3D path planning, in Proceeding of the 11th World Congress on Intelligent Control and Automation. 2015, IEEE: Shenyang, China.

4 Mohammadreza Radmanesh, et al., Overview of Path-Planning and Obstacle Avoidance Algorithms for UAVs: A Comparative Study. Unmanned Systems, 2018. 6(2): p. 13.

5 Noreen, I., A. Khan, and Z. Habib, Optimal Path Planning using RRT* based Approaches: A Survey and Future Directions. International Journal of Advanced Computer Science and Applications(IJACSA), 2016. 7: p. 11.

6 Jun, M. and R. D'Andrea, Path Planning for Unmanned Aerial Vehicles in Uncertain and Adversarial Environments, in Cooperative Control: 
Models, Applications and Algorithms, S. Butenko, R. Murphey, and P.M. Pardalos, Editors., Springer, Boston, MA: United States. p. 95-111.

7 Analyzer, C.A.-S.R., 'UAV' search results analysis: Research Areas Categories (1999-2019), in Clarivate Analytics - Web of Science, U.s.r.a.R.A.C. (1999-2019), Editor. 2020, Clarivate Analytics. 